\title{
Le rôle de médiation des bibliothèques patrimoniales en milieu universitaire
}

\section{The Mediation Role of Heritage Libraries Within a University Setting \\ La función de mediación de las bibliotecas patrimoniales en el ámbito universitario}

\section{Sarah de Bogui}

Volume 54, numéro 4, octobre-décembre 2008

URI : https://id.erudit.org/iderudit/1029188ar

DOI : https://doi.org/10.7202/1029188ar

Aller au sommaire du numéro

Éditeur(s)

Association pour l'avancement des sciences et des techniques de la documentation (ASTED)

ISSN

0315-2340 (imprimé)

2291-8949 (numérique)

Découvrir la revue

Citer cet article

de Bogui, S. (2008). Le rôle de médiation des bibliothèques patrimoniales en milieu universitaire. Documentation et bibliothèques, 54(4), 257-264.

https://doi.org/10.7202/1029188ar
Résumé de l'article

La médiation - terme rassembleur pour certains et fourre-tout pour d'autres - désigne l'ensemble des activités favorisant l'interaction entre collections et usagers. Dans le milieu des bibliothèques patrimoniales universitaires, cette mission acquiert une densité particulière puisque les collections constituent en elles-mêmes un matériau d'étude. « Anti-naturelle », la médiation est née de la nécessité de retisser le lien avec le patrimoine, un lien bouleversé par les événements qui ont agité la société québécoise au fil de son histoire. S'intéresser à la médiation n'est d'ailleurs pas seulement s'intéresser au contexte qui l'a vu naître : c'est aussi prendre la mesure d'une nouvelle forme de culture avec sa population, ses codes et son territoire. C'est enfin explorer les différents chemins qu'elle peut prendre concrètement et réfléchir sur ses fonctions plurielles.
Tous droits réservés (c) Association pour l'avancement des sciences et des techniques de la documentation (ASTED), 2008
Ce document est protégé par la loi sur le droit d'auteur. L'utilisation des services d'Érudit (y compris la reproduction) est assujettie à sa politique d'utilisation que vous pouvez consulter en ligne. 


\title{
Le rôle de médiation des bibliothèques patrimoniales en milieu universitaire
}

\author{
SARAH DE BOGUI \\ Chef de bibliothèque \\ Bibliothèque des livres rares et collections spéciales \\ sarah.de.bogui@umontreal.ca
}

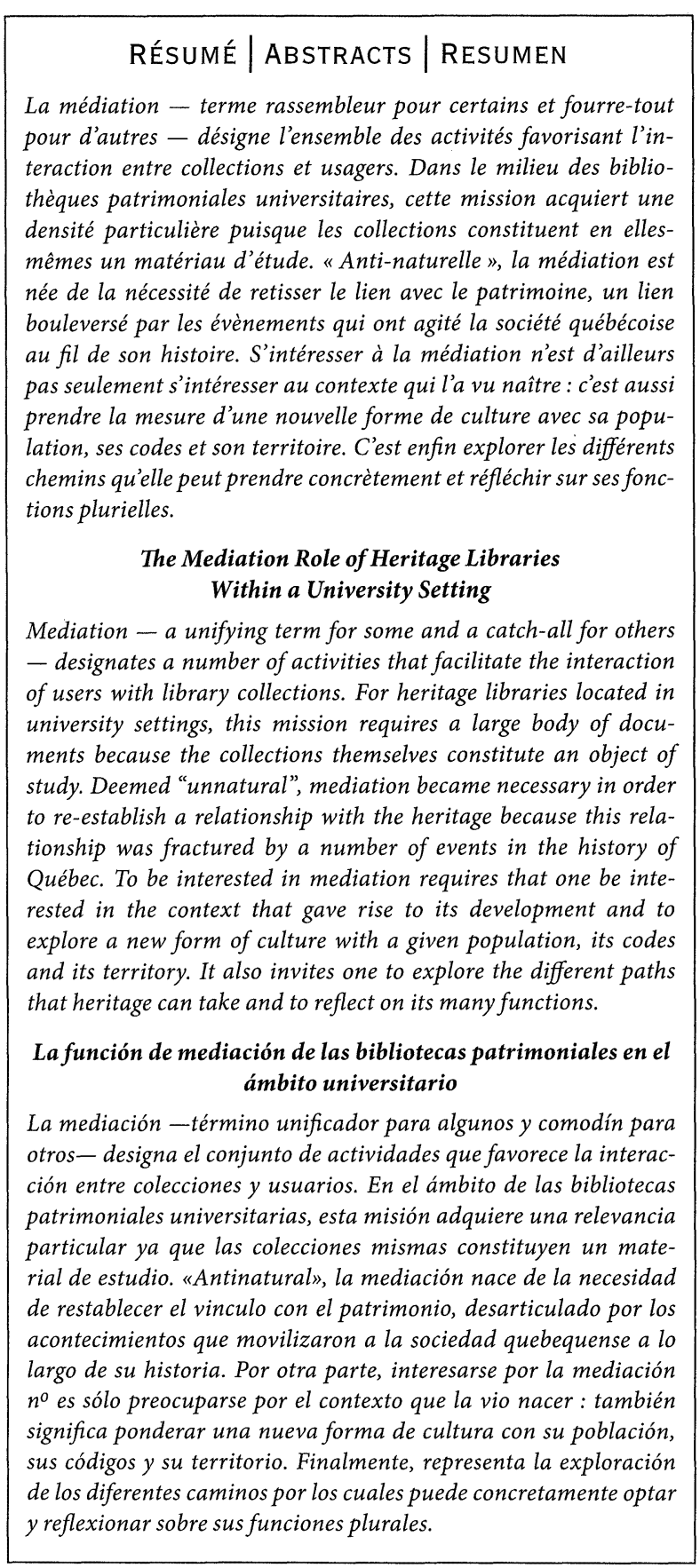

«De manière caractéristique, les premières bibliothèques universitaires sont des salles d'ouvrages de référence enchaînés à leurs pupitres."

Pallier et Poulot 2000, 133

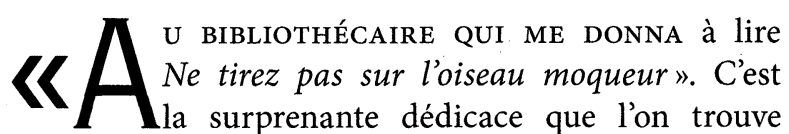
en ouverture d'un roman de Michael Connelly ${ }^{1}$. Voilà une forme rare et pour le moins remarquable de reconnaissance envoyée par un usager à un membre de notre profession. Nous ne savons rien de l'identité de ce bibliothécaire mais, en revanche, nous savons que ce qui lui vaut cet hommage est le rôle qu'il a joué pour l'auteur. En mettant en relation l'usager Connelly et le roman Ne tirez pas sur l'oiseau moqueur, le bibliothécaire a accompli une mission essentielle de la profession : la médiation. Dans le domaine des bibliothèques patrimoniales, cette mission possède une qualité plus dense encore puisque les documents qui y sont conservés sont perméables à deux jeux de lecture distincts qui en font des objets culturels polysémiques ${ }^{2}$. Michel de Certeau distingue ainsi deux formes de culture $(1974,290)$ : celle qui «permane " et «celle qui s'invente». Le message du document est la forme de culture qui "permane», qui reste statique dans le temps. En revanche, l'interprétation du message ou du document va varier selon les regards qui se poseront dessus : c'est la forme de culture «qui s'invente». Le patrimoine conservé suscite régulièrement des images funèbres où on les compare à des tombeaux organisés 3 . Pourtant, le patrimoine est bien vivant dans la mesure où il continue d'alimenter une culture en cours de création. Bien sûr, encore fautil, d'une part, avoir connaissance de ce patrimoine et, d'autre part, avoir la vision d'une culture où il trouve une nouvelle place. La phrase de Valéry est célèbre où, dans une allégorie du Musée de l'Homme de Paris, s'adressant à ses visiteurs, il s'écrie : "Il dépend de toi que je sois tombe ou trésor. " Dans les bibliothèques, les

\footnotetext{
1. Connelly, Micháel. À genoux. Paris : Éditions du Seuil, 2008.

2. Nous reprenons ici l'analyse de la lecture faite par Michel de Certeau $(1974,302)$ : «... le sens d'un texte est l'effet des procédures interprétatives appliquées sur la surface de ce texte. Aussi la permanence des textes est-elle un élément secondaire par rapport à la disparité des manières de les lire, puisque celles-ci affectent de significations hétérogènes les mêmes objets culturels."

3. En témoignent - parmi d'autres exemples - de nombreuses pages de l'ouvrage de Marc Guillaume, La politique du patrimoine (Éditions Galilée, 1980).
} 
professionnels sont un fil d'Ariane tendu devant les visiteurs, lequel peut les mener jusqu'au trésor : c'est ça, la médiation.

Lobjet de cet article n'est pas tant de faire une liste des activités de médiation connues, qui sont le fait des professionnels, qu'une réflexion sur les implications profondes de la médiation et des enjeux qui l'entourent. Une première étape consistera à démêler, grossièrement tout au moins, la manière dont s'articulent médiation, culture et patrimoine dans le milieu des bibliothèques universitaires. On abordera également la question des cadres généraux dans lesquels s'inscrivent habituellement les activités de médiation : réseaux, échanges et politiques. Et pour finir sur une note pragmatique - car la pratique est finalement le quotidien de tous - nous réfléchirons sur des exemples d'action de médiation et sur leur mise en place.

\section{Vous avez dit médiation ?}

Il est une question quon aborde peu en matière de médiation. Pourtant, la question na rien de rhétorique. Elle est la suivante: Pourquoi la médiation est-elle apparue ou, mieux, pourquoi la médiation s'est-elle peu à peu imposée comme une nécessité dans le domaine des bibliothèques patrimoniales?

Pour répondre à la question, nous devons nous livrer à une enquête historique afin de remonter à une époque où le livre, pas encore objet de patrimoine, était essentiellement objet de culture. Et auparavant, il nous sera bien sûr essentiel de revenir sur la définition du concept de médiation.

\subsection{La médiation}

La médiation est un concept qui s'applique à de nombreux domaines et qui trouve des significations aussi pointues que vagues. C'est donc très scolairement que, devant tant de pistes, nous allons commencer par prendre notre point d'ancrage dans un bon vieux dictionnaire. Celui que nous avons choisi4 énonce ceci : «1. Entremise destinée à mettre d'accord, à concilier ou à réconcilier des personnes, des partis. 2. Le fait de servir d'intermédiaire; ce qui sert d'intermédiaire. » Nous allons garder le terme "réconcilier" pour plus tard. Celui qui nous intéresse au premier chef, pour éclairer le type de médiation dont nous souhaitons parler ici, est « intermédiaire ». Et pour rétrécir un peu le - vaste champ de cette réflexion, nous allons nous en tenir aux trois domaines de médiation qui nous touchent de près : la médiation culturelle, la médiation du livre et la médiation du patrimoine. Ce qui demeure un programme pour le moins ambitieux...

La médiation culturelle englobe un vaste périmètre, dont les nombreux acteurs ne cessent de redéfinir les frontières. Afin de ne pas engager notre réflexion dans

4. Le Petit Robert: Dictionnaire de la langue française, nouvelle édition de juin 1996. d'interminables méandres, nous retiendrons cette définition proposée par la muséologue Élisabeth Caillet (1995, 23) : "Les compétences du champ de la médiation culturelle sont celles des professionnels [...] qui ont en charge l'accueil et l'information des publics [...] ». Que la générosité de cette définition ne soit pas prise à la légère : s'il est si complexe de borner cette activité, c'est bien justement parce que le tournant "orientation-client» pris par les sociétés occidentales élargit sans cesse le spectre des actions de médiation. La médiation culturelle, dont nous parlerons désormais, aura pour points de repère les quatre éléments suivants : l'institution culturelle, l'activité professionnelle, le service d'information et enfin le public-usager. En gardant à l'esprit que les actions de médiation culturelle doivent s'inscrire à l'intersection de ces quatre pôles, il devient plus facile de sorienter dans les discours. Dans le domaine du livre, la définition de la médiation senrichit d'une notion que nous avions mise de côté un peu plus tôt, c'est-à-dire : la réconciliation. Ainsi, alors que la médiation culturelle se définit fréquemment dans un environnement où les ambitions sont avant tout informationnelles, la médiation du livre, quant à elle, revendique avec ardeur ses ambitions interventionnistes. On lira par exemple sous la plume de Allouche $(2007,74)$ que la médiation du livre est une " [...] démarche professionnelle ou citoyenne qui consiste à organiser des rencontres autour des écrits, et des livres en particulier, en sollicitant la participation active des bénéficiaires. Pour cela, elle met en place une dynamique de liens ternaire (médiateur / écrits / médian) à travers un projet qui vise une égalité capacitaire de fait vis-à-vis de l'écrit, pour contribuer au pouvoir de lire de tous et réduire la distance sociologique entre les "non-lecteurs" ou les lecteurs potentiels et l'offre des institutions culturelles». On voit ici que le rôle de médiateur ou d'intermédiaire du professionnel n'est plus neutre mais vise à réconcilier deux réalités qui sont en opposition : la réalité sociale et la réalité institutionnelle. Sans aller jusquà rejoindre un objectif aussi volontaire, la médiation patrimoniale se situe cependant à mi-chemin entre cette vision de la médiation et une vision plus neutre telle que celle rapportée plus haut. En effet, le «document patrimonial» désigne un objet considéré comme suranné que seuls les historiens se plaisent encore à fréquenter et qui n’appartient plus à la caste enviée de ces livres, journaux ou autres articles intégrés aux réflexes de recherche des usagers du XXIe siècle.

Avant de poursuivre, faisons un bref rappel de ce que sont la «bibliothèque patrimoniale » et le «document patrimonial». Par bibliothèque patrimoniale, on entend une bibliothèque dont le mandat est de conserver et diffuser des documents patrimoniaux. Traditionnellement, est considéré comme patrimonial un document "rare, ancien ou précieux» (Desgraves, 1982). Par ailleurs, le terme "patrimoine " vient du mot "pater" qui, en latin, veut dire «le père ». On doit donc également lire dans le mot "patrimoine " la notion de filiation et de transmission (Poulot, $2006: 155$ ). Aujourd'hui, 
la définition de «document patrimonial» s'est ainsi élargie pour désigner plutôt le document «que lon conserve ou que l'on achète dans l'objectif d'une conservation à long terme" (Mouren, 2007 : 27). Pour clore cette courte présentation de la notion abondamment discutée de patrimoine des bibliothèques 5 , finissons sur cette très belle phrase des auteurs Chastel et Babelon $(1994,101)$ : «[...] dans toute société, le patrimoine se reconnaît au fait que sa perte constitue un sacrifice et que sa conservation suppose des sacrifices. "

Une forme d'interventionnisme est donc requise en bibliothèque patrimoniale pour réduire le fossé qui s'est creusé entre documents et lecteurs, car rappelons-le, ce document, en devenant patrimonial, est devenu objet muséologique. Or, «Lobjet est un lieu de rencontre entre des perceptions: il faut apprendre à regarder, à écouter. Il n'y a pas de lecture immédiate, spontanée, automatique." (Caune, 2006 : 147).

\subsection{Livre, culture, patrimoine}

Comme l'indique Élisabeth Caillet, "la question de laccès à la culture est à lorigine de la notion de médiation» (1995: 15). On ne peut donc faire léconomie d'une exploration de l'évolution de la notion de culture pour comprendre de quelle façon elle a engendré un rapport "médiatisé » aux documents patrimoniaux. Pour cela, il faut retourner au temps où savoir, culture savante et culture scientifique rimaient avec culture classique.

De nombreux auteurs soulignent la solidité et la récurrence du lien entre révolution politique et révolution culturelle ${ }^{6}$. Ainsi, en France, la Révolution française, par le rejet des traditions élitistes de l'aristocratie, a entraîné la mise en place des premières institutions culturelles publiques (musées, bibliothèques) et permis de développer la notion de "patrimoine" (Poirier et Wallon, $2000: 45$; Cabannes et Raffin, 2000 : 186).

Au Québec, deux évènements politiques majeurs ont entraîné un réajustement de la perception de la culture. Jusquà la Conquête, lélite intellectuelle canadienne française s'est efforcée de recréer les pratiques ayant eu cours en France (Falardeau, 1981: 40). À partir de la Conquête, le rejet de cette élite par les Anglais conduit à sa ruralisation, non seulement géographique - elle s'installe hors des zones urbaines - mais également intellectuelle (Lemire, 1981). Ceci, combiné à la montée de la bourgeoisie et des sciences techniques, conduit la culture québécoise à se distancer de la culture classique, héritée d'une vision humaniste et européenne du monde. Après 1840 , la culture classique - et ses raffinements constitue même une infraction au "code du bon campagnard» (Lemire, 1981: 79) alors que la culture canadienne française se vit plutôt « comme le moyen privilégié d'instaurer la patrie intérieure » (Lemire, 1981: 64). C’est

5. On lira également à ce sujet l'ouvrage dirigé par Jean-Paul Oddos (1997)

6. Citons pêle-mêle : Jean Caune, Babelon et Chastel, Dominique Poulot, Élisabeth Caillet, Julia Kristeva, Hanna Arendt, Michel de Certeau, etc. aux alentours de cette période que le discours national est exalté à travers le développement d'une culture littéraire, politique et artistique propre aux francophones $\mathrm{du}$ Canada et profondément soucieuse de revendiquer ses spécificités tant vis-à-vis des Anglais que des Français et des Américains. La culture de lélite intellectuelle canadienne française se caractérise donc finalement par une remarquable hybridité : en surface une culture classique, mâtinée du sens pratique propre à la culture bourgeoise et, en profondeur, une culture nationaliste ancrée dans la ruralité. Pour les notables appartenant à lélite, accumuler les preuves de la culture nationale devient alors une forme de passe-temps qui conduit parfois au développement de véritables collections documentaires7. Contrairement aux objets muséaux dont la «perte d’usage a, dans la plupart des cas, précédé la "patrimonialisation" $[. .]$.$» (Béghain, 2000: 30$ ), le livre conserve intacte la nature de son message. Sa patrimonialisation procède davantage du contexte de collection et de la mutation du contexte culturel dans lequel il s'inscrit et qui vient enrichir le message originel de sens nouveaux.

Le second évènement politique dont les répercussions ont été ressenties jusque dans la perception de la notion de culture, remettant en cause, par le fait même, la notion de patrimoine, est cette Révolution quon appelle tranquille. Elle a bien sûr provoqué d'intenses bouleversements, y compris dans la manière de considérer léducation et donc le savoir. Au cours des années 1960, la culture classique est remise en cause de manière virulente, jusquà perdre le combat face à l'onde de choc du Rapport Parent (Corbo et Couture, 2000). Cette culture classique cesse également doccuper sa place dans les programmes universitaires (pensons à la disparition des cours en histoire des disciplines, l'histoire de la médecine, par exemple). Être scientifique, être savant, prend alors une nouvelle signification. Le rapport au livre, alors, change évidemment. Issues des volontés patientes d'une élite intellectuelle dans laquelle la population ne se reconnait plus, les collections érudites ne jouissent plus de la même faveur. Mais bientôt, comme le dit Hansen ${ }^{8}$ : "Ce que le fils désire oublier, c'est ce dont le petit-fils cherche à se souvenir", et ces collections sont exhumées comme mémoire de la culture nationale. Le piège serait alors de sacraliser ces pièces et ces ensembles au risque de les pétrifier et de les transformer en momies du savoir. Aujourd'hui, la médiation a donc pour fonction de réinsérer ces objets dans les pratiques d'études modernes, non plus en tant que matériau de construction d'une culture fonctionnelle, mais en tant que matériau de construction d'une culture mémorielle. Ainsi, à l'image d'un Bardo Thodöl9 dédié aux âmes des documents, la médiation montre la voie vers la réincarnation...

\footnotetext{
7. On pensera sans peine à Philéas Gagnon, à Louis-François-Georges Baby, à l'abbé Verreault, à Pierre-Joseph-Olivier Chauveau, etc.

8. Cité par Babelon et Chastel in La notion de patrimoine, p.110.

9. Livre tibétain des morts décrivant les étapes successives à franchir entre l'instant de la mort et celui de la réincarnation.
} 


\section{Les cadres de la médiation}

«La culture est communication ». Cette affirmation de Jean Caune (2006: 51) ouvre la porte aux questionnements soulevés par la réflexion sur les cadres de la médiation. Comme toute culture, la culture de la médiation possède en effet ses indigènes - les publics - , ses codes linguistiques - les outils - , et sa délimitation géographique - les politiques.

\subsection{Les publics des bibliothèques patrimoniales}

La relation entre documents et usagers des bibliothèques patrimoniales semble sépanouir entre deux bornes aux antipodes l'une de l'autre. De manière imagée, on pourrait définir cet espace comme un vaste territoire dont les frontières se situent entre l'inutile et le sacré.

Le personnel des bibliothèques est en première ligne lorsqu'il s'agit d'interaction entre document et usager. Le premier contact peut donc jouer un rôle primordial. En effet, les bibliothèques patrimoniales souffrent parfois de leur image pourtant dépassée de prestige et délitisme. Les néophytes et les âmes impressionnables hésitent bien souvent à pénétrer dans des lieux où le charme discret du luxe ajoute à l'appréhension de demander la consultation d'ouvrages dont l'ancienneté projette déjà une aura de terreur sacrée. Il faut impérativement démystifier les bibliothèques patrimoniales. Un personnel accessible, accueillant et didactique, sans être condescendant, est la clé d'une relation décomplexée entre l'usager craintif et les collections.

Dans un autre cas de figure, le public, étant bien souvent spécialiste dans son domaine, peut avoir de la difficulté à saisir les apports possibles d'un recours aux professionnels. Tout bibliothécaire de référence disciplinaire a en mémoire des anecdotes sur ce genre de situation. En bibliothèque patrimoniale, la difficulté réside dans le fait que la référence couvre en réalité des activités mal connues qui se rapportent davantage à l'offre de référence offerte dans les services d'archives que dans les bibliothèques. Ainsi, on le sait, les bibliothécaires disciplinaires vont, par exemple, offrir à leurs usagers des séances de formation à l'utilisation des bases de données spécialisées ou encore aider au montage de bibliographies d'articles et de monographies sur un sujet donné. Là, se situe la différence majeure avec l'offre faite par le bibliothécaire patrimonial qui concentre plutôt ses recherches sur les sources primaires et tertiaires. Prenons l'exemple d'un usager faisant une recherche sur la représentation de l'Enfer dans la littérature du XVIIe siècle. Alors que le bibliothécaire disciplinaire va permettre au chercheur de connaitre les bases utiles à la recherche et de trouver une liste d'articles et d'ouvrages sur cette question, la tâche du bibliothécaire patrimonial consistera à trouver dans ses collections du XVIIe siècle des illustrations de l'Enfer Et s'il existe une ou plusieurs bibliographies spécialisées des livres illustrés de cette période, le bibliothécaire ne manquera pas de les faire connaître à son usager. Ainsi, alors que chez le bibliothécaire disciplinaire, le nerf de la guerre est sa parfaite connaissance des bases de données, ouvrages de référence spécialisés et autres sources d'information, chez le bibliothécaire patrimonial, le nerf de la guerre est sa parfaite connaissance des collections qu'il a à sa disposition et des sources d'information sur les documents patrimoniaux. Cependant, le bibliothécaire patrimonial est encore, dans le regard d'un certain nombre d'usagers, la figure pointue et moustachue du rat de bibliothèque qui connaît par cœur chaque titre de sa collection. Et, sauf pour aller chercher « vous savez, l'édition de Machin commentée par Truc...", il ne saurait apporter de valeur ajoutée au travail du chercheur que par l'agréable diversion qu'offre sa conversation érudite.

Qu'on n'interprète pas à tort ces quelques lignes: la peinture de ces extrêmes est avant tout le prétexte à décrire en partie les spécificités de la relation professionnelle à l'usager en bibliothèque patrimoniale. Que lon comprenne bien qu'entre les deux se situe la majorité du public: de plus en plus, on voit se développer des programmes conjoints de recherche où les professionnels des bibliothèques patrimoniales sont sollicités - adieu crainte sacrée - pour apporter leur expertise - adieu docte mais inutile rongeur.

\subsection{Les outils de la médiation}

Le travail de médiation s'appuie, en bibliothèque comme en tout autre établissement de culture ou de savoir, sur des outils qui sont, d'une part, les outils d'analyse stratégique, et d'autre part, les outils de communication.

La connaissance des publics auxquels on s'adresse et l'analyse de ses besoins propres sont des éléments essentiels à la base de toute initiative de médiation. D’ailleurs, la muséologue Élisabeth Caillet (1995: 70) souligne: "[...] il importe de refaire lanalyse de ses publics à chaque fois que l'on construit ou reconstruit son projet culturel». Et qui sont les publics des bibliothèques patrimoniales? Des universitaires en grande majorité, étudiants aussi bien que professeurs-chercheurs, mais aussi quelques étudiants du secondaire, du grand public érudit ou curieux et, enfin, des professionnels des métiers du livre, artistes et commerçants. Lorientation intellectuelle de ce public est aussi majoritairement marquée par les sciences humaines : histoire, littérature, histoire de l'art, architecture... Cet aspect du profil n’a guère changé depuis que "l'honnête homme » a commencé à se livrer à létude et à la recherche. En revanche, ce qui a changé, ce sont les attentes de ce public : fini le temps où l'accès au sanctuaire de la bibliothèque constituait la récompense ultime du visiteur. Les bibliothèques patrimoniales se sont ouvertes, aussi largement que leurs consœurs disciplinaires et du secteur public, et les usagers se sont découverts clients. Ainsi, il n'est pas envisageable de faire l'impasse sur les services de référence et de reproduction. 
Un autre facteur a beaucoup contribué à augmenter les attentes : le développement du Web. Grâce à Internet, il est devenu possible, non seulement d'offrir des contenus numériques, mais aussi de mettre en vitrine son offre de service. Tout en restant chez lui, n'importe quel usager peut aller voir ce qui se fait dans d'autres pays et sur d'autres continents. Cette mondialisation de l'offre culturelle est un moteur non négligeable dans lévolution des produits et des services qu'il faut développer.

De plus en plus, loffre de service, on l'a dit, est disponible directement sur le Web. Ce dernier est un formidable agent de séduction, mais aussi un outil de médiation dans le sens où il est à la fois instrument de proximité et d'interactivité. Les services offerts par les bibliothèques patrimoniales sont nombreux : formation à la recherche spécialisée, sensibilisation à la conservation des documents, collections de signets, revues érudites, bulletins spécialisés, répertoires et inventaires, contenus axés sur la bibliophilie, expositions virtuelles, etc. Lautre grand volet Web de loffre de service en bibliothèque patrimoniale est bien sûr la bibliothèque numérique. Le défi lancé par Google a trouvé des échos jusque dans le salon du citoyen lambda, dûment informé par les médias généralistes. Une bataille s'est engagée avec comme compétiteurs l'Europe puis la Francophonie. Comme le mentionnait récemment Jack Kessler ${ }^{10}$, un observateur des bibliothèques numériques, la moindre petite bibliothèque patrimoniale possède à présent son projet de diffusion sur le Web. Ce serait déchoir que de navoir rien sur la table. Ce qui engendre deux autres problèmes dont le premier est bien connu des professionnels : l'infobésité. Car, comment aujourd'hui, avec la prolifération de projets, est-il possible de savoir ce qui est mis à disposition sur la toile ? Rassembler cette information pour constituer une bibliothèque mondiale de ressources est un nouvel enjeu auquel il est, pour l'instant, difficile de trouver une solution toute faite. Le second problème, d'ordre local celui-là, est le rétrécissement progressif du potentiel de numérisation des bibliothèques patrimoniales québécoises. En effet, en-dehors de manuscrits qui sont des pièces uniques, les collections patrimoniales sont principalement constituées d'imprimés. Les premiers imprimés québécois étant apparus en 1764 , tout ce qui constitue les fonds dits " anciens » (c'est-à-dire antérieurs au XIX ${ }^{\mathrm{e}}$ siècle) provient majoritairement d'Europe. Et comme on l'a évoqué plus haut, l'Europe a beaucoup progressé dans les travaux de numérisation de son patrimoine. Lautre grand joueur est $B A n Q$, dont lobjectif est de numériser le patrimoine imprimé québécois. Enfin, les États-Unis, loin dêtre en reste dans cette marche à la diffusion numérique, ont développé une offre dont l'ampleur fait le régal des chercheurs. On a vu que l'analyse des besoins du public était un fondement essentiel à la mise en place d'actions de médiation. La médiation, consistant notamment à offrir

10. "Smalltown digital libraries». In FYI France ejournal, 15 septembre 2008. URL: http://www.fyifrance.com/Fyarch/fy080915.htm. un accès élargi aux documents, entre autres par le moyen d'Internet, il faut donc éviter de tomber dans le piège de la redite et de réaliser, avec un train de retard, ce qui se fait ailleurs, et parfois en mieux. On comprend qu'une fine analyse stratégique est incontournable lorsqu'il s'agit de se lancer dans un programme de médiation, qu'il soit réel ou virtuel. Cette analyse comprend également les choix à faire en matière de promotion culturelle. Bien sûr, les actions de médiation ne peuvent faire l'économie d'un travail de communication. Le plan de communication est un outil qui vient en amont de la médiation, de la même manière que l'analyse stratégique vient en aval. Les actions menées dépendront des publics cibles et orienteront le choix des médias et du discours de promotion. Enfin, il est un dernier outil indispensable : lévaluation de laction de médiation.

\subsection{Les politiques de médiation}

Comme les matriochkas, ces poupées russes qui s'emboîtent les unes dans les autres, les politiques de médiation s'inscrivent dans un environnement plus vaste que représente la politique patrimoniale, elle-même produit de la politique culturelle. Au Québec, la politique culturelle est issue du métissage entre pratiques française, anglaise et américaine (Colbert, 2008). Le ministère de la Culture ${ }^{11}$, né en 1961 sous le nom de ministère des Affaires culturelles, s'inspire en effet directement du schéma français de "l'État architecte " faisant figure de "monarchie culturelle» (Colbert, 2008: 7). Quant au Conseil des arts et des lettres du Québec, créé en 1992, on ne saurait lui nier au moins quelques liens de parenté avec le Arts Council of England ${ }^{12}$, lequel a fait de l'Angleterre un "État mécène " (Colbert, $2008:$ 7-8). Enfin la figure américaine de "l'État facilitateur» (Colbert, $2008: 8$ ), où prime lencouragement au développement grâce au mécénat, se fait de plus en plus sentir dans les pratiques québécoises. Et de la même manière que, de poupée en poupée, chaque modèle n'est que la reproduction à moindre échelle du modèle précédent, le système mixte de la politique culturelle québécoise se reproduit jusqu'au maillon ultime de la politique de médiation. En effet, en plus des ressources budgétaires allouées par l'établissement universitaire, les bibliothèques patrimoniales peuvent se voir octroyer des subventions gouvernementales spécifiques grâce aux programmes d'aide développés au sein du ministère de la Culture comme au Conseil des arts et des lettres. Et il nest pas rare de voir des projets de médiation se réaliser grâce à l’appui de mécènes. À léchelle de la bibliothèque, les politiques de médiation se développent donc sur les bases d'une politique culturelle mixte plébiscitée à léchelle nationale, tout en s'inscrivant dans le champ des ressources offertes par l'université.

\footnotetext{
11. Pour des raisons pratiques, nous nommerons simplement «ministère de la Culture » ce qui se nomme actuellement en réalité «ministère de la Culture, de la Communication et de la Condition féminine ".

12. Anciennement : Arts Council of Great Britain.
} 
Mais avec l'arrivée du numérique, les politiques de médiation doivent tenir compte des nouveaux enjeux tels que la mondialisation, le Web 2.0 ou encore la législation. La mondialisation, nous l'avons évoquée plus haut, a un impact direct sur les axes de développement des actions de médiation. Le Web 2.0, en misant sur l'interaction entre les utilisateurs et les technologies, oblige à revisiter certaines actions de médiation, mais permet également d’en inventer de nouvelles ${ }^{13}$. Enfin, lappétit augmentant et les technologies évoluant, la question du respect du droit d'auteur prend de plus en plus de place dans la réflexion professionnelle.

Ainsi, lorsque les acteurs de bibliothèques patrimoniales souhaitent se doter d'un outil, il est important de prendre en compte les publics et l'environnement institutionnel, certes, mais également de considérer l'environnement culturel et l'environnement technologique.

\section{Activités de médiation}

\subsection{Niveaux de la médiation}

$\mathrm{Au}$ XIII siècle, l'abbé parisien Hugues de SaintVictor écrivit dans son Didascalicon: "Lordre de la lecture consiste à chercher d'abord la lettre, ensuite le sens, enfin la pensée ». Il voulait signifier par là que la compréhension des textes passe par plusieurs étapes, correspondant à différents niveaux de lecture : la lettre qui vise à déchiffrer le texte, le sens qui vise à comprendre grammaticalement le texte et enfin la pensée qui vise à comprendre les idées émises par l'auteur.

On pourrait, de la même manière que Saint-Victor, appliquer cette approche scolastique de la lecture aux différents niveaux de médiation qui peuvent être atteints grâce aux activités développées en bibliothèques. Ainsi le premier niveau, la lettre, couvre toutes les activités de médiation qui permettent d'appréhender la relation physique au document - les caractéristiques matérielles des documents sont d'une grande importance dans les collections patrimoniales. Le second niveau, le sens, couvre les activités de médiation permettant d'appréhender globalement le contenu et le contexte de production du document. Enfin le troisième niveau, la pensée, couvre les activités de médiation qui permettent d'interpréter le document, c'est-à-dire de l'exploiter comme matériau de recherche ou source primaire d'information dans un cadre scientifique. Les activités de médiation offrent, bien sûr, plusieurs facettes, ce qui fait en sorte que selon ses récepteurs, les niveaux de lecture vont varier. Si l’on pense, par exemple, à une activité populaire telle que l'exposition, les trois niveaux sont couverts : les visiteurs de premier niveau s'intéresseront uniquement à lapparence physique des documents ou objets exposés,

13. Consulter à ce sujet les communications du colloque Patrimoine numérique et médiation culturelle organisé le 22 octobre 2008 par l'Agence régionale pour le livre et l'écrit en Aquitaine (ARPEL). URL: http://arpel.aquitaine.fr/spip. php?article100001991. les visiteurs de second niveau liront les cartels ${ }^{14}$ et les panneaux pour connaitre le contenu du document et le mettre en contexte et les visiteurs de troisième niveau entreront en contact direct avec le document afin de l'intégrer comme sujet ou comme référence à des recherches universitaires ou scientifiques.

\subsection{Accès aux documents}

Parmi les activités de médiation, l'accès aux documents est celle qui prime avant toute autre puisquelle répond à la mission essentielle des bibliothèques. En cela, la bibliothèque patrimoniale, souvent perçue comme un musée de par ses collections et sa vocation de conservation, se distingue indiscutablement des établissements muséaux où l'accès aux objets est restreint. Dans quel musée un visiteur pourrait-il se présenter et demander à voir l'un des tableaux conservé dans les réserves ${ }^{15}$ ? C'est pourtant ce qui est pratiqué dans les bibliothèques patrimoniales, car au-delà de la valeur artistique, vénale ou historique d'un objet, c'est sa nature «lisible» qui lui donne sa puissance. Empêcher la lecture d'un livre, sous prétexte de sa préciosité, revient à le faire mourir puisque son message ne peut être délivré. Mais, comme l'ont souligné les auteurs Cabannes et Raffin (2000: 193) «[...] il est évident qu'il faut un passeur capable de produire les bons outils permettant d'accéder aux codes: cest là que se place limmense travail de médiation qu'entreprennent aujourd'hui les bibliothécaires [...]». Ainsi, la production d'outils de recherche spécialisés (catalogues de collections notamment) concourt à la mission de médiation entreprise dans les bibliothèques patrimoniales.

\subsection{Expositions}

L'une des autres activités populaire de médiation déjà évoquée plus haut est l'exposition. L'exposition est un moyen qui, on l'a vu, permet d'atteindre plusieurs niveaux de lecture en même temps. En conséquence, il faut aborder les documents à exposer sous des angles multiples: du physique, du contenu et du contexte. L'une des difficultés importante à laquelle on se trouve confronté, lorsqu'on expose des livres, est leur inflexible banalité. Le livre est un objet que le quotidien a fini par faire passer dans la trivialité et sa forme récurrente (un bloc de feuilles de papier enserrées dans une couverture) est d'un manque doriginalité navrante pour qui songe à la mettre en valeur. Les livres d'artistes ${ }^{16}$ et les livre-

14. Petit carton explicatif accompagnant chaque document ou objet exposé. On y trouve au minimum le titre, l'auteur et la date de production de l'œuvre exposée. Les cartels sont plus ou moins détaillés selon le concept de l'exposition.

15. Les réserves sont des salles d'entreposage habituellement sécurisées où le climat (température, humidité, luminosité) est contrôlé. L'ensemble de ces dispositifs ayant pour but de garantir la protection et la conservation à long terme des objets qui s'y trouvent.

16. Livre ayant la vocation d'être en lui-même une œuvre d'art, que ce soit grâce à son iconographie, sa typographie, sa forme, son papier, etc. 
objets $^{17}$ ont su apporter enfin un peu de soulagement au découragement des bibliothécaires et conservateurs, mais on ne peut, malgré tout, se contenter de n'exposer que ces livres-là ! La scénographie ${ }^{18}$ des expositions est donc un élément primordial dans la présentation des documents, car le premier niveau de médiation reste l'aspect matériel des objets exposés. Le second niveau de médiation, c'est-à-dire le contenu du document et sa mise en contexte, ne constitue pas un obstacle. En revanche le troisième niveau qui, rappelons-le, vise à interpréter le document, est autrement plus complexe. L'enjeu consiste ici à développer un propos cohérent qui sera porté par les documents exposés. Il s'agit alors de sélectionner les documents en fonction du sujet de l'exposition. Pour ce faire, il faut se livrer à un exercice dont les termes peuvent sembler bien ésotériques, mais qui seront pourtant compris de tous les membres de la profession: écouter les collections. Écouter les collections, c'est-à-dire comprendre leurs orientations, leurs forces et leurs faiblesses, c'est entendre sélever peu à peu le propos de lexposition et son articulation. Inscrits dans ce propos, c'est tout naturellement que les documents pourront se laisser interpréter.

\subsection{Conférences}

D’autres actions de médiation sont couramment entreprises dans les bibliothèques patrimoniales, offrant d'autres modes d'accès et de compréhension des documents. Les conférences sur l'histoire du livre ou sur tout thème pouvant être illustré par des documents issus des collections (évènements historiques, mouvements de la pensée, évolutions techniques, etc.) remportent généralement de francs succès. Une variante de ces conférences, où le propos est plus didactique que magistral et où surtout le contact direct avec les documents pour son apport pragmatique est privilégié, existe depuis de nombreuses années en France sous le terme de "classes patrimoine ${ }^{19}$. Destinées aussi bien aux adultes qu'aux enfants du primaire ou du secondaire, elles sont passées dans les pratiques éducatives du secteur patrimonial.

\subsection{L'exploitation scientifique : ultime consécration}

Montrer les livres nest pas tout: ce n'est en fait que le début. Car si lon montre le livre, c'est pour qu'enfin, un jour, il trouve son chercheur. Pour remplir son office, un tableau, une sculpture, une œuvre plastique, doivent être regardés. Un objet usuel utilisé. Un livre lu. En bibliothèque patrimoniale, comme en bibliothèque publique ou disciplinaire, un livre qui n'est pas utilisé est

17. Objet dépassant généralement la forme traditionnelle du livre mais répondant à la même vocation.

18. La scénographie consiste à mettre en scène et en valeur les objets exposés, notamment par le décor. On en parle parfois comme de la "médiation de l'espace ».

19. Dans le cas des classes patrimoine, le patrimoine va aussi jusqu'à se déplacer dans les classes. un livre qui ne sert à rien. C'est pourquoi le travail de médiation tend finalement vers un grand objectif : faire du livre conservé un objet détude. Et comme le souligne Lecoq (2000: 65): "[...] l'exploitation scientifique des documents patrimoniaux n'est que l'aboutissement d'un long processus, fait de mesures discrètes et patientes." C'est pourquoi la réalisation d'un tel objectif demande une bonne connaissance des fonds, du milieu universitaire et des réseaux de la recherche. Et comme tout entremetteur professionnel, le bibliothécaire doit parfois utiliser quelques subterfuges et tenter de nombreuses combinaisons avant que se produise le mariage parfait. On en revient, encore une fois, à l'absolue nécessité de connaître son public et ses besoins. Avec la médiation, il devient possible de voir se transformer des documents ou des collections en objets de recherche, de publications, de subventions et, finalement, de rayonnement scientifique. Les bibliothèques patrimoniales ont cette particularité d’offrir à leurs usagers plus que des ustensiles : elles offrent aussi de la nourriture. Les bibliothèques universitaires québécoises l'ont bien compris et s'ingénient de plus en plus à développer des collaborations fructueuses avec la communauté universitaire. À titre d'exemples, citons le travail de recherche sur la Collection Napoléon à l'Université McGill ${ }^{20}$, la mise en valeur $\mathrm{du}$ fonds ancien à l'UQAM ${ }^{21}$ ou encore l'exploitation des livres savants de l'Université de Montréal ${ }^{22}$. Chacun de ces projets, soutenu par des subventions, a donné lieu à un travail de recherche scientifique et a généré publications imprimées et numériques, conférences, colloques. Ainsi, dans des domaines d'intérêts variés, les collections patrimoniales apportent la preuve d'une contribution concrète à l'avancement de la connaissance.

\section{Conclusion}

La mission des bibliothèques universitaires saffiche clairement comme un soutien à l'enseignement et à la recherche. Or cette mission proclamée fait léconomie d'un aspect des collections documentaires oublié par beaucoup : non seulement "soutien" mais également «matière » à l'enseignement et à la recherche. En laissant de côté ce pan de la mission des bibliothèques universitaires, le milieu a peut-être commis une bévue que paient depuis longtemps de nombreuses bibliothèques patrimoniales en milieu universitaire. Les propos de Lecoq (2000: 62) tenus dans le contexte des bibliothèques universitaires françaises s'appliquent tout aussi bien aux bibliothèques québécoises : "Au mieux exploitées d'une façon purement utilitaire et parfois sans égards, au pire considérées comme un accessoire décoratif relevant d'une forme de standing, ces collections [patrimoniales] semblent échapper aux enjeux que se donne l'université. "

\footnotetext{
20. http://digital.library.mcgill.ca/napoleon/francais/index.htm

21. http://www.livresanciens.uqam.ca/

22. http://www.bib.umontreal.ca/CS/livre-savant/intro.htm
} 
Le travail de médiation s'efforce de remédier à cet état de fait. Et finalement, il devient possible de considérer que, quel que soit le niveau de médiation atteint avec les différents publics, les actions entreprises à cet égard dans les bibliothèques patrimoniales remplissent le rôle de rouages discrets mais essentiels à l'avancement des loisirs, de l'éducation et de la science.

Ainsi, loin des anciens lieux sombres et poussiéreux plus fréquentés par les rongeurs et les érudits atteints de la goutte que par la lumière du jour, les bibliothèques patrimoniales des $\mathrm{XX}^{\mathrm{e}}$ et XXIe siècles se sont transformées en institutions résolument modernes, ouvertes et engagées dans leur société. $\odot$

\section{Sources consultées}

Allouche, Abdelwahed. 2007. Les médiations dans les bibliothèques publiques. Bulletin des bibliothèques de France. 52 (6) : 71-77.

Babelon, Jean-Pierre et André Chastel. 1994. La notion de patrimoine. Paris : L.Lévi.

Béghain, Patrice. 2000. Quels fondements pour une politique de conservation? Bulletin des bibliothèques de France. 45 (4): 29-31.

Cabannes, Viviane et Raffin, Anne. 1997. Le secret. In Le musée et la bibliothèque, vrais parents ou faux amis. Sous la direction d'Anne-Marie Bertrand. Paris : Bibliothèque publique d'information, Centre Georges Pompidou : 181-193.

Caillet, Élisabeth. 1995. À l'approche du musée : la médiation culturelle. Lyon: Presses universitaires de Lyon.

Caune, Jean. 2006. Culture et communication : convergences théoriques et lieux de médiation. Grenoble : Presses universitaires de Grenoble.

Certeau, Michel de. 1974. La culture au pluriel. Collection 10 :18. Paris : Union générale d'éditions.
Chourrot, Olivier. 2007. Le bibliothécaire est-il un médiateur? Bulletin des bibliothèques de France. 52 (6) : 67-71.

Colbert, François. 2008. Les éléments des politiques culturelles. Séminaires de management culturel de la Chaire de gestion des arts Carmelle et Rémi-Marcoux. URL : http://www.gestiondesarts.com/index.php ?id=62.

Corbo, Claude et Jean-Pierre Couture (dir.). 200o. Repenser l'école : une anthologie des débats sur l'éducation au Québec de 1945 au rapport Parent. [Montréal] : Presses de l'Université de Montréal.

Desgraves, Louis. 1982. Le patrimoine des bibliothèques : rapport au directeur du livre et de la lecture. Paris : Ministère de la Culture.

Falardeau, Jean-Charles. 1981. Savoirs savants et savoirs populaires. In Cette culture qu'on appelle savante. Sous la direction de François Dumont. Montréal : IQRC et Léméac : 35-46.

Lamizet, Bernard.1999. La médiation culturelle. Paris : L'Harmattan.

Lecoq, Benoît. 2000. Quelques réflexions sur les bibliothèques universitaires et leur patrimoine. Bulletin des bibliothèques de France. 45 (4) : 61-65.

Lemire, Maurice. 1981. Savoir et pouvoir : le cas du Bas-Canada. In Cette culture qu'on appelle savante, sous la direction de François Dumont. Montréal : IQRC et Léméac : 63-79.

Mouren, Raphaële (dir.). 2007. Manuel du patrimoine en bibliothèque. Paris : Éditions du Cercle de la Librairie.

Oddos, Jean-Paul (dir.). 1997. Le patrimoine : histoire, pratiques et perspectives. Paris : Éditions du Cercle de la Librairie.

Pallier, Denis et Poulot, Dominique. 1997. Les collections. In Le musée et la bibliothèque, vrais parents ou faux amis ? Sous la direction de Anne-Marie Bertrand. Paris : Bibliothèque publique d'information, Centre Georges Pompidou : 131-16o.

Poulot, Dominique. 2006. Une histoire du patrimoine en Occident. Le nœud gordien. Paris : Presses universitaires de France.

Riegl, Alois. 1984. Le culte moderne des monuments : Son essence et sa genèse. Espacements. Paris : Éditions du Seuil.

Six, Jean-François. 1995. Dynamique de la médiation. Paris : Desclée de Brouwer. 